\title{
AN INSIGHT INTO THE HOUSEKEEPING PRACTICES OF SRI LANKAN HEALTHCARE FACILITIES IN PRIVATE SECTOR
}

\author{
S.S.C GINTHOTAVIDANA ${ }^{1} \&$ K.G.A.S. WAIDYASEKARA ${ }^{2}$ \\ 1,2University of Moratuwa, Katubedda, Sri Lanka \\ 1sscgvidana@gmail.com, 2anuradha@uom.lk
}

\begin{abstract}
The inferior performance in housekeeping services has contributed to distinguishable levels of patient dissatisfaction. Therefore, similar to primary processes like medical treatment in healthcare sector same attention should be paid on secondary processes such as housekeeping services by the management. Moreover, the knowledge on housekeeping services is critical to plan and design proper housekeeping mechanisms in healthcare facilities. Therefore, this paper aims to explore the nature of housekeeping services in order to design proper housekeeping processes for private healthcare facilities in Sri Lanka. This research follows a qualitative research approach to examine the current housekeeping practices by adopting case study strategy. Semi structured interviews were conducted with seven respondents of the selected three cases from the private sector and direct observation method was used to better understand the housekeeping procedures. The results revealed that even though the bed capacity belongs to three groups, the housekeeping practices remain almost similar and the housekeeping in hospitals is essentially based on infection control and hygiene rather than aesthetical appearance compared to other facilities. Furthermore, the key differences such as absence of key designations such as upholsterers, painters, wall washers and catering team in the hierarchy and the absence of floor plans and area responsibility plans in the housekeeping divisions in Sri Lankan context were determined by comparing the research findings with the literature sources.
\end{abstract}

Keywords: Healthcare Facilities; Housekeeping; Processes; Infection Control

\section{Introduction}

The researches point out that an unclean healthcare environment can contribute to the increase in the risk of infections to patients (Dancer, 2004). Boyce (2007) highlights that the factors such as the ability of microbes to remain on the surfaces of the hospital areas, how often the pathogens get contaminated to the highly touched surfaces in the hospital and the number of pathogens in the hospital areas contribute to the transmission of infections in a healthcare setting. The environmental hygiene level of healthcare facilities is ensured through a chain of interconnected activities, which are critical to the infection control (Malik, Cooper and Griffith, 2003). As explained by Sherlock et al. (2009), the adequate removal of pathogens and microorganisms from the healthcare environments is crucial to minimize the spread of diseases, which contributes to overall hygiene. As indicated by the findings of Andaleeb (2001), the hospitals should always have a clean and pleasant environment in a proper order to satisfy their customers. Sevin (2018) points out that the housekeeping services provided by a particular hospital is different from the provision of medical treatment, which is its primary service, but essentially has a positive effect on the quality of medical service provision, patient satisfaction and attitudes of the patients.

There is a noticeable shortage of the housekeeping staff who are competent in human resource management, financial management and housekeeping operational skills, which create barriers in innovations related to housekeeping, and this leads to a failure of appreciation in housekeeping service aspects such as people, finance and operations (Anthonisz, 2014). Due to the high level of focus on the primary processes, the activities, which are supportive to the primary process such as housekeeping receives a low level of attention as they do not directly contribute for the income generation in healthcare environments (Horrevorts, et al., 2018). There is a distinguishable satisfaction gap of the hospital occupants related to the cleanliness and housekeeping performance of Sri Lankan Hospitals (Senarath et al., 2013). As there are very limited researches conducted in relation to the housekeeping practices of Sri Lankan healthcare facilities, there is a need to explore the 
nature of housekeeping services in order to design proper housekeeping processes for the local healthcare facilities. Therefore, the aim of this paper is to examine the current housekeeping practices in Sri Lankan private sector healthcare facilities.

\section{Literature Review}

The satisfaction of customers relating to housekeeping services can be identified as significant in determining the customer loyalty (Kandampully and Suhartanto, 2000). The American Hospital Association (2006) highlights that the housekeeping hierarchy of a large hospital consists of important key positions such as Executive housekeeper, Assistant housekeeper, Painters and wall washers, Linen room staff, Upholsterers, Housekeeping supervisors, Maids and Porters.

Further, within the housekeeping organisational structure in ward management as explained by May and Suckley (2005), the facilities manager supports the care team by supervising the ward housekeeper and the cleaning team by assigning relevant duties such as cleaning, catering and linen management. The ward sister also manages the care team and their support staff simultaneously with the facilities manager. Both the facilities manager and the ward sister should effectively plan and communicate for the proper functioning of the ward housekeeping team.

The American Hospital Association (2006) classifies the major housekeeping activities done in healthcare environments as indicated in Figure 1.The housekeeping activities have been classified as daily cleaning activities, which are essential to day to day operation of the hospital environment, periodic cleaning activities, which are performed to maintain all the elements in the environment in a proper order, and upkeep maintenance performed to enhance the lifetime of various components of the hospital setting.

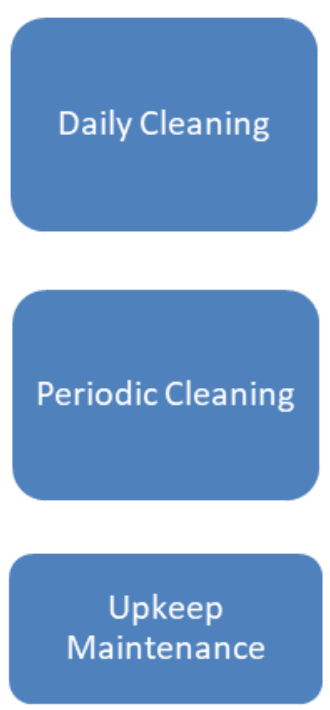

- All public floors, such as reception room, entrance

halls,corridors, elevators, stairways

- Patients' rooms and wards

- Emergency rooms, treatment rooms

- Dressing rooms, rest rooms,lavatories

- Service rooms

- Supply rooms

- Window washing

- Wall cleaning

- Floor waxing and polishing

- Furniture washing and polishing

- Rug and carpet shampooing

- Upholstery cleaning

- Other work done biweekly, weekly, fortnightly, monthly

- Painting

- Furniture refinishing

- Drapery making or repairing

- Repair and replacement of furniture and furnishings

Figure 1, Housekeeping Activities (Source: American Hospital Association)

Furthermore, Wiggins (2014) described the five (05) typical areas of cleaning and housekeeping services as general cleaning, waste disposal, janitorial work, window cleaning and deep cleaning. Moreover, the author classifies the cleaning methods used in healthcare sector into major five (05) categories as physically clean, chemically clean, bacteriologically clean, entomologically clean and osmologically clean. Specifically, entomologically clean means the absence of harmful insects such as pests and the absence of surface dust and debris on all surfaces of the healthcare facility. Moreover, the term osmologically clean refers to the absence of any organic or inorganic matter which emits an 
odour and this is the level of cleanliness needed in intensive care units, operation theatres and food processing industries. When a particular area is osmologically clean, the probability of transmitting harmful pathogens are reduced to a great extent.

The general areas of pathogen accumulation in a hospital environment as indicated by the Ministry of Health and Family Welfare (2015), are the bed, bedside table, bed linen, bed frames, bed rail, floor, pillow, telephones, call bell, bedside locker, curtains, stationary, mattress, television, pressure machine, key board, stethoscope, sink, tv remotes, couch, door handle, thermometer, bathroom, faucet handle, toilet commode, tables, dustbin, and window frames. Ministry of health and Family Welfare (2015), further mentions that hospital cleaning should be carried as per the following guidelines presented in Table 1.

Table 1 : Area cleaning of healthcare facilities

\begin{tabular}{|c|c|c|}
\hline Area category & Frequency of cleaning & Level of cleaning \\
\hline High risk areas & $\begin{array}{l}\text { Once in } 2 \text { hours with spot } \\
\text { cleaning as based on the } \\
\text { requirement }\end{array}$ & $\begin{array}{lr}\text { Cleaning } & \text { and } \\
\text { intermediate } & \text { category } \\
\text { disinfection } & \end{array}$ \\
\hline Moderate risk areas & $\begin{array}{l}\begin{array}{l}\text { Once in } 4 \text { hours } \\
\text { cleaning bith spot } \\
\text { requirement }\end{array} \\
\text { requ }\end{array}$ & $\begin{array}{l}\text { Cleaning and low category } \\
\text { disinfection }\end{array}$ \\
\hline Low risk areas & $\begin{array}{l}\text { Once or twice in a shift with spot } \\
\text { cleaning }\end{array}$ & Cleaning only \\
\hline
\end{tabular}

(Source: Ministry of health and Family Welfare)

Furthermore, Wiggins (2014) mentions that the cleaning and housekeeping equipment and materials are of a wide variety such as floor buffers, robotic controls, backpack vacuum cleaners, ionators, water poles, blade driers and floor scrubbing machines. Additionally, the author highlights that the cleaning chemicals can be ranging from acidic to alkaline solutions, which are categorised as detergents, sealants, and solvents. Jones (2008) explains that an executive housekeeper should possess important documents such as division of work document, area responsibility plan, floor plan layouts and the criteria for workloads.

\section{Research methodology}

This research follows a qualitative approach by adopting case study strategy in order to obtain the necessary information on the housekeeping practices of Sri Lankan healthcare facilities. Three (o3) Cases (A, B, C) were selected from the private healthcare institutions located in Colombo district with seven (07) respondents (AR1, AR2, AR3, BR1, BR2, BR3, CR1). The Case selection was done based on judgement subjected to the resource limitations and access limitations. The Case A was a large scale healthcare facility ( 400 beds), the Case B was of medium scale (26o beds) and the Case C was of small scale (6o beds). All the Cases have received ISO 9001 certification and possess both in house and outsourced housekeeping service staff. The Case study boundary of this research can be identified as the healthcare sector and the unit of analysis can be highlighted as the housekeeping services in the healthcare sector. The data collection was carried out through the semi structured interviews using an interview guideline as the data collection tool. Direct observation was also used as a data collection tool in order to obtain a proper understanding of the housekeeping procedures. Other data collection methods were not considered because the suitability of those methods did not match to the research outcome. The respondents covered multiple designations related to the housekeeping service management as indicated in Table 2. 
Table 2: Summary of Interview Respondents

\begin{tabular}{|c|l|l|c|}
\hline Case & Respondent & \multicolumn{1}{|c|}{ Designation } & Years of Experience \\
\hline \multirow{4}{*}{ A } & AR1 & Executive Housekeeper & 31 years \\
\cline { 2 - 4 } & AR2 & Assistant Housekeeper & 18 years \\
\hline \multirow{3}{*}{ B } & AR3 & Senior operations manager (outsourced) & 20 years \\
\cline { 2 - 4 } & BR1 & Chief Operating Officer & 15 years \\
\cline { 2 - 4 } & BR2 & Assistant manager (Quality Assurance) & 7 years \\
\hline BR3 & Facilities Manager (Quality Assurance & 4 years \\
\hline & Division) & 20 years \\
\hline
\end{tabular}

The collected data were analysed using cross case analysis technique by focusing on important areas in relation to the existing practices of housekeeping services in the local context.

\section{Data Analysis and findings}

The existing practices of the housekeeping departments in the healthcare sector was identified using the interviews focused on the three (03) Cases A, B and C. Findings were discussed under main headings of structure, work processes and significance of services in the housekeeping department.

\subsection{THE STRUCTURE OF THE HOUSEKEEPING DEPARTMENT}

The initial section of the semi structured interview guideline was focused on obtaining a proper insight into the background of the housekeeping function in a healthcare environment. As explained by all of the respondents, the housekeeping department of a healthcare setting consists of linen room, laundry and cleaning sections. The Executive Housekeeper of Case A (AR1) stated, "There are outsourced companies like outsourced pest control, gardening and cleaning under housekeeping". The Chief Operating Officer of Case B (BR1) explained, "There is a Central Sterile Supplies Division (CSSD) linked to housekeeping". The Facilities Manager of Case C (CR1) mentioned, "There is a housekeeping store under the housekeeping department".

According to the respondents $\mathrm{AR} 1, \mathrm{AR} 2$ and $\mathrm{AR} 3$ of Case $\mathrm{A}$, the executive housekeeper oversees housekeeping operations and under him the housekeeping supervisors, linen assistants and operational level staff is prevalent. While explaining the hierarchy of Case B, BR1 mentioned that, he is the Chief Operating Officer (COO), who oversees the housekeeping function and other functions of the hospital. The housekeeping manager is under the $\mathrm{COO}$ and there are five (05) housekeeping supervisors reporting to him. Under the housekeeping supervisor there are about 45 janitors who are at the operational level of the hierarchy. In the Case $\mathrm{C}$, the housekeeping department is a sub section of the facilities management department and it has different functions such as laundry, cleaning and stores. The respondent CR1 explained the hierarchy of the department as, "The facilities manager is the head of the facility department and there is an executive housekeeper under him who looks after the linen and laundry division. There are operational and administration staff under the executive housekeeper". The designations explained in the literature such as upholsterers, painters, wall washers and catering team were not mentioned by the respondents. Even though, the designations of the top management and housekeeping staff in each case is slightly different, the functions and responsibilities of the positions remained similar in all the Cases. Basically, this hierarchy is formed around the different housekeeping functions in a healthcare setting such as cleaning, linen and laundry, stores and additional outsourced functions such as gardening and pest controlling. By considering all those inputs a concept organisational structure of a housekeeping division developed for a Sri Lankan private sector healthcare environment is illustrated in Figure 2. 


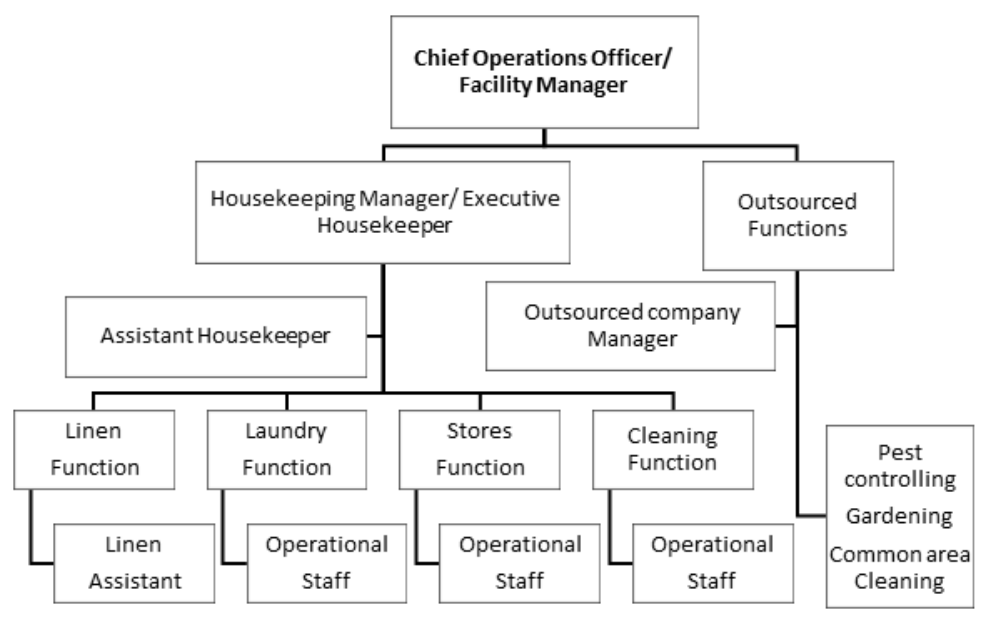

Figure 2, organizational structure of the housekeeping department

\subsection{WORK PROCESSES OF THE HOUSEKEEPING DIVISION IN HEALTHCARE SECTOR}

According to the interviews based on the selected Cases, four (04) main work processes were identified within the housekeeping department. They were Cleaning process, Linen and Laundry process, Waste Management process and Stores Management process. Moreover, the supportive processes such as documentation, chemical usage and testing were also visible in the housekeeping division. The following sub sections discuss each process in a detailed manner.

\subsubsection{Cleaning Process}

All the respondents stated that, cleaning is a vital function undertaken by the housekeeping department and the work done through the cleaning division has a high level of value addition to maintain the hygiene of the hospital environment. The Executive Housekeeper of Case A (AR1), explained that the cleaning frequencies in relation to Case A are twice a day for patient rooms, ward cleaning once a week and heavy duty cleaning in annual or once in 6 month intervals. In relation to Case B, the Chief Operating Officer (BR1) stated that the daily cleaning is done three (O3) times a day in the morning, mid-day and in night time for critical areas such as Intensive Care Units (ICU) and Heart Command Centre by performing normal housekeeping activities such as mopping, sweeping and washroom cleaning. The deep cleaning is done once a week and scrubbing is done in monthly intervals. Similar arrangement could be found in Case $\mathrm{C}$ as well. The cleaning frequency of critical areas in Case A was lower than Case B and C due to the high level of patient occupancy and staff shortages. The cleaning staff were wearing shoes, gloves, uniforms, caps and carried a linen bucket in all of the Cases, which demonstrated adherence to the personal safety and hygiene standards and specific cleaning intervals around the day was found in all three cases. Another interesting finding was, the allocation of a special colour coding system for cleaning equipment used in the specific areas in the hospital. As highlighted by the respondent AR1, the colour blue for rooms and public areas, red for washrooms, yellow for ICU and theatres and green for cafeteria and kitchen is being used. The Facilities Manager of Case B (BR3) explained the existing practice for equipment separation as, "There are separate mops used for infected areas, which are not colour coded but labelled and separated". The colour coding system has been effectively used in Case $\mathrm{C}$ to segregate the cleaning equipment, which is somewhat similar to Case A as, blue (corridors, Temporary Surgery Unit, office areas, ETUs, radiology offices, all public areas), red (washrooms, bathrooms, showers, toilets, wash basins and bathroom floors), yellow (surgery ward, ICU, labour room, neo natal ICU, theatre, lab areas, isolation areas, mortuary, Phlebotomy) and green (kitchen and cafeteria). In Case A, Case B and Case C, there are different cleaning functions, which are performed by the outsourced staff but commonly those areas were of a non-critical nature. 


\subsubsection{Linen and laundry process}

The linen and laundry process was present in all three (03) Cases. The Assistant Housekeeper of Case A (AR2) stated, "All linen is collected twice a day by linen and laundry staff and special procedures are followed in collecting, washing and issuing linen". As mentioned by AR1 and AR2, the chemicals used for the laundry operation are detergent, neutralizer, liquid sour, alkaline builder duty liquid, chlorine and bleaching powder. The operation of the laundry function in Case B has some similar aspects with the Case A. Additionally, the Assistant Quality Assurance Manager of Case B (BR2) stated, "After washing the linen, for the sterilizing purpose they send it to the autoclave, which is linked to CSSD". The activities done in the laundry are quite similar in Case A, B and C. Moreover, Facilities manager of Case C (CR1) stated, "germed or contaminated items are going through two wash cycles", which is different from other Cases. Additionally, the matching of the soil linen to clean linen is carried out in the linen room area in Case $\mathrm{C}$ along with issuing of uniforms to staff members three (03) times a day. Further, CR1 highlighted, "Fabric Softener, slash destroyer for oils, Oxy Splash for bleaching, emulsifier and detergent are used as laundry chemicals".

\subsubsection{Waste management process}

The waste management function is inevitably linked to the housekeeping department because it is connected to the cleaning function. As explained by AR1, there are two main types of waste in a relation to Case A, named as hazardous and non-hazardous waste. The infectious waste, clinical waste, sharp items, pharmaceutical waste and radioactive waste can be considered as hazardous waste and paper, plastic, and biodegradable waste are non-hazardous waste. The respondent AR1 mentioned, "The waste is collected using a World Health Organisation (WHO) accepted colour coded bin system in operating theatres, ICUs and other areas where the hazardous waste is generated". The colour coding system uses orange for plastic waste, red for glass waste, black for general non-infectious waste, yellow for infectious waste and green for biodegradable waste. The colour codes in Case B are different from Case A in few areas, which are identified as yellow with red stripe (sharp waste), Orange 1 (recyclable plastics), Orange 2 (other plastics). The colour codes of Case A and C are of a similar nature. The food waste is sold to a private company to prepare pig meals. Paper, plastic polythene and, other waste is directly handed over to the municipal council. The sharp waste boxes and hazardous waste are incinerated in all three (03) Cases and the other types of waste are disposed through the involvement of the Municipal Council. The additional work processes related to the housekeeping division apart from the above mentioned four (04) key work processes are discussed in the following sections in an extensive manner.

\subsubsection{Stores management process}

The stores function is aligned with the housekeeping operations in Case $\mathrm{C}$ and it was not present in the other two Cases. Respondent CR1 mentioned, "The items in the stores are hand towels, toilet rolls, garbage bags, disposable cups and housekeeping chemicals". As elaborated by CR1, the goods are purchased to the stores through issuing a Purchase Request Form (PRF) and audits and checks for the stores are performed by the main office of the hospital.

\subsubsection{Documentation process}

The inputs from the respondents of all the Cases suggested that documentation is an important aspect within the housekeeping service management of a healthcare facility. The documents used in Case A as explained by AR1, AR2 and AR3, included housekeeping checklists dedicated to different areas in the hospital, attendance registers of staff, Inventory records of linen, furniture and furnishings and standard operating procedures for the housekeeping department. In the Case B, the documents maintained are similar to Case A, but the use of checklists are expanded. BR1 explained, "Room cleaning checklist, critical area cleaning checklist and a washroom cleaning checklist are maintained by supervisors, janitors and the housekeeping manager for daily operations". Furthermore, BR2 stated that, monthly calendar, documents for daily cleaning and deep cleaning, housekeeping manual, infection control manual and Joint Commission International (JCI) standards are the documents used in Case B. The performance measures listed for the housekeeping department of Case B are proper management of issuing chemicals by authorized persons, dilution of chemicals according to ratios, 
proper distribution of cleaning equipment with proper replacements, on the job training, proper maintenance of records on housekeeping department and timely maintenance and monitoring of all the areas of the hospital. The documents used in Case $\mathrm{C}$ were similar to Case $\mathrm{A}$ as per the information provided by CR1. Even though, the literature suggest that the executive housekeeper should maintain floor plan layouts and area responsibility plans those were not available in any of the Cases.

\subsubsection{Chemical usage and testing processes}

All the respondents emphasised that the proper chemical usage and testing ensures the efficient provision of housekeeping services. The respondent AR1 mentioned that, the chemicals mainly used in the housekeeping department are toilet bowl cleaner, all purpose or surface cleaner, air freshener and glass cleaner with zero ammonia. The chemicals used in Case B according to the standard operating procedure document were sanitizer, all-purpose cleaner, glassware cleaner, air freshener, tile cleaner, toilet bowl cleaner, Clorex bleach, GC 100 (to be used at the garbage disposal and outside), bleach powder, Calcium Hypochlorite (TCL),floor sealer and floor striper. Respondent CR1 mentioned the same types of chemicals used by the other two Cases for the housekeeping functions. The biological testing or culture testing is performed in theatre, ICU, Surgical rooms and Surgical wards in Case A and Air conditioning plate tests are done for microbiological growth in Case B. The culture test is done in the laboratory before a critical operation such as kidney transplantation in Case C. By considering the testing practices of all Cases, it is evident that a high level of focus is given for the culture tests in Cases A and B, when compared to Case C. As Case C only performs culture testing before critical operations, the risk of contamination during minor surgeries and other medical procedures might not get captured more often.

\subsection{SIGNIFICANCE OF HOUSEKEEPING SERVICES IN HEALTHCARE SECTOR}

The housekeeping services in healthcare sector are fundamentally different from other facilities such as shopping malls, offices, hotels, educational institutions etc. Importantly, respondent AR1 stated, "if you think about the hospitals, we have to go to the infection control and hygienic side more, because the appearance and the cleanliness of course should be there, but more than that, infection control should be there". Respondent AR2 stated a different viewpoint regarding healthcare sector housekeeping services as, "The customer in a hospital is different than other facilities and they have a different set of expectations". The respondent BR1 had a statement, which supported the idea provided by AR2 as, "Mainly we are dealing with the patients and when we are dealing with the patients, our hospital and facilities should be in really high standards at any given time". CR1 supported this idea by stating, "The doctors, patients and other parties also pay more attention to the safety, hygiene and hazard control”, highlighting the occupant expectations in a hospital. Therefore, it is evident that the healthcare housekeeping is at a critical and a distinguishable stage when compared with the housekeeping divisions of the other facilities.

\section{Discussion}

The level of hygiene in a healthcare environment can be escalated through the efficient planning of housekeeping services. The results revealed that the hierarchy of Sri Lankan healthcare housekeeping divisions are quite similar to the literature findings, which amalgamates different functions such as laundry, linen handling, stores, cleaning, pest controlling and gardening. Even though, the literature highlights that the positions such as upholsterers, painters, wall washers and catering team in the housekeeping hierarchy, these were not included in the Sri Lankan context because those functions are handled specifically by the maintenance division or outsourced contractors, in the healthcare setups. Therefore, it is evident that the Sri Lankan housekeeping divisions are not competent enough to include the maintenance activities into their scope. The catering is usually a separate function related to the hospitality services. The findings highlight that the critical areas of each healthcare organisation, which are cleaned more frequently and thoroughly, are handled by the in house housekeeping team to maintain a high level of hygiene, even though some non critical areas are handled by outsourced staff. linen and laundry function in each hospital is contributing to the infection control through thorough disinfection of infected linen items which are responsible for the spread of pathogens. A key feature in the waste management of all healthcare settings is the colour 
coded waste separation methods. Sharp waste and infectious waste are new categories of waste, which are only identifiable in healthcare organisations. The documentation and chemical handling functions can be identified as supportive housekeeping functions in all of the cases, which ensures the proper performance of housekeeping activities in a healthcare environment. Floor plan layouts and area responsibility plans were not available in Sri Lankan healthcare housekeeping divisions because the management hasn't dedicated enough time to develop those complex housekeeping planning tools. The stores management function was only present in one case, which indicates that generally that function is connected to the purchasing division of a healthcare setup. The results indicate that the healthcare housekeeping function is different from the other types of facilities because the end customer is the patient, who expects a high level of hygiene and infection control in the target environment.

\section{Conclusions and Way Forward}

This paper focuses on the existing housekeeping practices of the Sri Lankan private sector healthcare facilities. The literature findings provide a general idea on the housekeeping services. The findings of the qualitative study revealed information about the existing structure of housekeeping department including the key designations, the work processes (cleaning, linen and laundry, waste management, stores management, housekeeping documentation and chemical handling and testing) and the significance of housekeeping services in healthcare sector. Even though the healthcare facilities were from different scales, the functions performed by the housekeeping divisions remained almost similar. It was also found that the healthcare sector focuses more on infection control and hygiene compared to other facilities based on the opinions of the respondents. Those results were discussed in comparison with the findings of the literature survey to identify the key differences in the practices. This research would be beneficial for the industry practitioners to gather detailed knowledge to properly manage the housekeeping divisions, to assist in the planning of housekeeping services, to develop new strategies to enhance customer satisfaction and to understand the critical nature of the healthcare housekeeping services. The facilities management practitioners who are new to the healthcare industry could also utilise the findings to determine the differences in housekeeping services in healthcare facilities compared to other organisations and adapt themselves to better manage the housekeeping services in healthcare sector to effectively generate cost savings. Finally, the paper looks at the current practices of housekeeping function in healthcare sector in order to develop a performance measurement model as the next step of the study which promotes service quality and efficiency of the healthcare facilities.

\section{References}

American Hospital Association. (2006). Manual of Hospital Housekeeping, American Hospital Association, Illinois.

Andaleeb, S. S. (2001), "Service quality perceptions and patient satisfaction: a study of hospitals in a developing country", Social Science and Medicine, Vol. 52 No. 9, pp. 1359-1370.

Andrews, S. (2013), Hotel Housekeeping, McGraw Hill Education (India) Private Limited, New Delhi.

Anthonisz, A. (2014), "Assessing the future of housekeeping operations in Dubai's five-star hotel industry - room for innovation?”, Worldwide Hospitality and Tourism Themes, Vol. 6 No. 4, pp. 352-361.

Boyce, J. M. (2007), "Environmental contamination makes an important contribution to hospital infection", Journal of Hospital Infection, Vol. 65, pp. 50-54.

Dancer, S. J. (2004), "How do we assess hospital cleaning? A proposal for microbiological standards for surface hygiene in hospitals", Journal of Hospital Infection, Vol. 56 No. 1, pp. 10-15.

Horrevorts, M., Terpstra, P. and Ophem, J.V. (2018), "Impact of cleanliness on the productivity of employees", Facilities, Vol. 36 No. 9/10, pp. 442-459.

Jones, T. J. (2008), Professional Management of Housekeeping Operations, John Wiley and Sons Inc, New Jersey.

Kandampully, J. and Suhartanto, D. (2000), "Customer loyalty in the hotel industry: the role of customer satisfaction and image", International Journal of Contemporary Hospitality Management, Vol. 12 No. 6, pp. 346-351.

Malik, R.E., Cooper, R.A. and Griffith, C.J. (2003), "Use of audit tools to evaluate the efficacy of cleaning systems in hospitals", American Journal of Infection Control, Vol. 31 No. 3, pp. 181-187.

May, D. and Suckley, L. (2005), "Ward housekeepers in mental health environments", Facilities, Vol. 23 No. 13/14, pp. 6o8620.

Ministry of Health and Family Welfare (2015), National Guidelines for Clean Hospitals, Ministry of Health and Family Welfare, New Delhi. 
Senarath, U., Gunawardhana, S. N., Sebastiampillai, B., Senanayake, A., Lekamge, S., Seneviratna, A. and Wijeratne, D. (2013), "Patient satisfaction with nursing care and related hospital services at the National Hospital of Sri Lanka", Leadership in Health Services, Vol. 26 No. 1, pp. 63-77.

Sevin, H. D. (2018), "Hotel Services in Hospitals", Journal of Tourism and Gastronomy Studies, Vol. 6 No. 1, pp. $451-459$.

Sherlock, O., O’Connell, N., Creamer, E. and Humphreys, H. (2009), "Is it really clean? An evaluation of the efficacy of four methods for determining hospital cleanliness", Journal of Hospital Infection, Vol. 72 No. 2, pp. 140-146.

Wiggins, J. M. (2014), "Housekeeping and Cleaning Services”, In Facilities Manager's Desk Reference, John Wiley \& Sons, West Sussex, pp. 437-456. 